\title{
GCU
}

Glasgow Caledonian

University

University for the Common Good

\section{An evaluation of mainstream type 2 diabetes educational programmes in relation to the needs of people with intellectual disabilities: a systematic review of the literature}

Maine, Andrew; Brown, Michael J.; Dickson, Adele; Truesdale, Maria

Published in:

Journal of Applied Research in Intellectual Disabilities

DOI:

10.1111/jar. 12544

Publication date:

2019

Document Version

Author accepted manuscript

Link to publication in ResearchOnline

Citation for published version (Harvard):

Maine, A, Brown, MJ, Dickson, A \& Truesdale, M 2019, 'An evaluation of mainstream type 2 diabetes

educational programmes in relation to the needs of people with intellectual disabilities: a systematic review of the literature', Journal of Applied Research in Intellectual Disabilities, vol. 32, no. 2, pp. 256-279.

https://doi.org/10.1111/jar.12544

\section{General rights}

Copyright and moral rights for the publications made accessible in the public portal are retained by the authors and/or other copyright owners and it is a condition of accessing publications that users recognise and abide by the legal requirements associated with these rights.

Take down policy

If you believe that this document breaches copyright please view our takedown policy at https://edshare.gcu.ac.uk/id/eprint/5179 for details

of how to contact us. 
An evaluation of mainstream type 2 diabetes educational programs in relation to the needs of people with intellectual disabilities: A systematic review of the literature

Andrew Maine ${ }^{1}$, Michael J. Brown ${ }^{2}$, Adele Dickson ${ }^{3}$, Maria Truesdale ${ }^{1}$

1. Edinburgh Napier University, Edinburgh Napier University School of Health and Social Care, 9 Sighthill Court, Edinburgh, UK EH114BN; 2. Queen's University Belfast, School of Nursing and Midwifery, Belfast, UK; 3: Glasgow Caledonian University School of Health and Life Sciences, Glasgow, Glasgow, UK

Corresponding author: Andrew.maine@napier.ac.uk 


\begin{abstract}
Background: Risk reduction and self-management programs for type 2 diabetes (T2D) are commonplace. However, little is known about their appropriateness for people with intellectual disabilities (ID). This review evaluates successful components and theoretical basis of interventions and preventions in relation to the needs of people with ID with or at risk of T2D.
\end{abstract}

Method: Characteristics of 23 randomised controlled trialled T2D educational programs were systematically assessed alongside the needs of people with ID, and evaluated in terms of study design and theoretical application.

Results: Successful components of programs align to the needs of people with ID. Further adaptations are required to ensure accessibility of materials and social support to enable reflection on illness perceptions and self-efficacy, as underpinned by Self-regulation and Social-cognitive theories.

Conclusions: Support is provided for further trials of self-management and preventative adaptations under development. Impact may be enhanced through preventions aimed at younger groups in educational settings.

\title{
1. Introduction
}

Diabetes is a global health concern, with 1.5 million deaths around the world attributed to the disease annually. A worldwide majority of $90 \%$ of people with diabetes have type 2 diabetes (WHO, 2016). Type 1 diabetes (T1D) is where insulin production is insufficient and is associated with an autoimmune disorder. Type 2 diabetes (T2D) is the instability of blood sugar levels due to the body's low or ineffectively used insulin, and is associated with 
lifestyle factors, for example obesity, lack of physical activity, and medication control (Scottish Public Health Observatory, 2013). Due to the high and increasing prevalence of the disorder, research has focussed on how T2D can be self-managed effectively.

Self-management involves various lifestyle adaptations, including diet, exercise, monitoring of blood sugar levels, foot care, and adherence to medication regimes. Barriers to self-management have been identified, including lack of diabetes knowledge (Davies et al. 2008, Yates et al., 2009), attitudes and expectations to exercise (Absetz et al., 2007; Jenum et al., 2006), and socioeconomic factors (Contento, Koch, Lee, and Calabrese-Barton, 2010; Utz et al., 2008). The importance of overcoming these barriers is emphasised through the increasingly severe cost implications of diabetes on care services. Diabetes UK reported a cost of $£ 23$ billion in 2010/2011, of which 8.8 billion was for T2D (Hex, Bartlett, Wright, Taylor, and Varley, 2012). This figure is projected to almost double by 2035. Given that lifestyle intervention programmes have been shown to reduce the risk of T2D by up to $60 \%$ (Gillies et al., 2007), research on enabling and sustaining self-management is important.

\subsection{Theory driven interventions}

The importance of giving strong theoretical basis to intervention programs is well documented (Gourland et al., 2016; Michie and Prestwich, 2010). Nutbeam and Harris (2010) provide a framework of models used in healthcare interventions, in which there are four major models aimed at lifestyle behavioural change: the Health Belief Model (HBM), the theories of reasoned action and planned behaviour (TRA, TPB), the transtheoretical (stages of change) model (TTM), and Social cognitive theory (SCT). Within the HBM model (Becker, 1974) there are 4 factors which affect the likelihood of action being taken: perceived susceptibility, perceived seriousness, perceived benefits, and perceived barriers. While 
changes in these beliefs have resulted in behavioural change, the model has been less successful in addressing long term, socially influenced health behaviours (Nutbeam and Harris, 2010). The TRA and TPB models (Ajzen and Fishbein, 1980, Ajzen, 1991) emphasises the importance of intention to act on behavioural change. These intentions are governed by attitudes and subjective norms, as well as perceived control in relation to situations. The TTM model (O'Hara, 1996) presents a descriptive model of readiness to change, which includes precontemplation, contemplation, determination, action, and maintenance. The model is useful in terms of identifying and adapting to the present needs of individuals, however it may have limited application in the clinical setting (Nutbeam and Harris, 2010). SCT (Bandura, 1977), is based on the relationship between an individual and their environment, emphasising the interaction between social influence and cognition. The model has been successful in informing education programmes aimed at behavioural change (Nutbeam and Harris, 2010). In addition to these models, Leventhal's Self-Regulatory Theory (SRT) has been applied to interventions by focussing on individuals' representations of their illnesses, thereby providing a basis for behavioural change (Taggart et al. 2015).

The common focus across all the models is the individual, with a focus on beliefs, attitudes and responses to social influences. While there is a large body of evidence regarding the efficacy of the models within the general population, little is known about their suitability for people with intellectual disabilities.

\subsection{Diabetes and people with intellectual disabilities}

The self-management of T2D can present significant challenges for people with intellectual disabilities (ID), including socio-economic inequalities (Emerson, 2005), limited support worker knowledge (Cardol, Rijken, and van Schrojenstein Lantman-de Valk, 2012b), 
restricted access to services (Dysch, Chung, and Fox, 2012), and poor living arrangements which contribute to sedentary lifestyles and poor diet (Melville et al., 2008). Health services further contribute to the situation by failing to make reasonable adjustments to care and support that takes account of and responds to the cognitive and communication impairments experienced by many of this population. When coupled with low health literacy in people with ID, there are significant consequences in relation to making healthy lifestyle choices (Emerson and Baines 2010). Further research is therefore required to more fully understand how T2D interventions can be developed to improve health and facilitate self-management for people with ID.

McVilly et al. (2014) undertook a systematic review of the research literature regarding the prevalence, incidence and impact of T1D and T2D in people with ID. The findings reflected a paucity of research in the area, with 13 studies focusing on prevalence and suggesting a mean prevalence of $8.6 \%$. The diversity in methodological approaches with these studies made incidence unclear. Three studies reported on self-management of diabetes (Cardol et al., 2012a; Hale, Trip, Whitehead, Conder, and Jenny, 2011). All were qualitative, and contained small sample sizes with unclear evidence of data saturation, making it difficult to draw conclusions regarding options and ways forward. The studies highlighted people with ID's poor understanding of the severity of their condition, frustration around dietary changes, confusion around what constitutes physical activity and wider psychosocial issues surrounding social support from carers.

A second review was conducted by Macrae et al. (2015). As with McVilly et al. (2014), variability was reported in the quality of prevalence studies. The paper also highlighted that many studies failed to report important demographic information, including the level of ID. This would have better explained variation in factors like carer support, and highlights the importance of balancing and recording baseline demographics in order to 
effectively highlight differences in subgroups. Macrae et al. (2015) concluded that in order to develop effective self-management strategies, research should be undertaken which explores the feasibility of adapting mainstream T2D educational programs to the ID population. There are currently no published studies on the effectiveness of T2D interventions for people with ID.

The aim of this review is therefore to systematically evaluate mainstream T2D interventions, with a view to informing the development of theoretically grounded interventions for the ID population. Studies included in the review were evaluated in terms of the quality of their design, with those that received a low rating discarded. Following review, those detailing theoretical models were evaluated in terms of quality and clarity of evidence. The models are critically analysed and discussed in the context of the current literature regarding the self-management of T2D in PWID.

\subsection{Systematic review questions}

1. What are the characteristics, including successful outcomes, of theoretically informed T2D self-management interventions, and what is the appropriateness of these characteristics for people with ID?

2. What is the methodological quality of these studies?

3. What is the quality of the evidence regarding the utilisation of theoretical models and their translation and application to intervention techniques? 


\section{Method}

This systematic review is reported following the Preferred Reporting Items for Systematic Reviews and Meta-Analyses statement (Liberati et al., 2009).

\subsection{Search Strategy}

A systematic, comprehensive approach was taken using the following electronic data bases: PsychINFO, Psychology and Behavioural Sciences Collection, CINAHL, MEDLINE and PubMed. Search strategies were unique to each database according to suggested search terms, for example through MESH or Thesaurus. Systematic reviews of public health interventions present challenges due to database indexing issues (Tacconelli, 2010). With this in consideration, search terms, sub-headings and filters were designed to be as broadly inclusive as possible. These included "Type 2 Diabetes" OR "Diabetes Mellitus" OR "Noninsulin dependent diabetes" AND "self-management" OR "self-care", with sub-headings protection and control and filters set to quantitative only, and clinical queries set to Therapy: high sensitivity. An additional search was conducted with the preceding terms AND “Intellectual Disabilities” OR “Learning Disabilities” OR “Mental Retardation”.

\subsection{Inclusion and exclusion criteria}

This review employed the following inclusion search criteria:

1) Type of paper: original, peer-reviewed research articles, excluding thesis, dissertations, books, reviews and government reports

2) Study Design: Quantitative studies employing randomized controlled trials (RCTs), 
3) Theoretical model: only studies which included and make explicitly referenced to a theoretical model were included.

The following were excluded:

1) Qualitative studies

2) Pseudo-experimental designs

3) Feasibility studies

4) Cross-sectional surveys

5) Follow-up studies,

6) Studies not published in English

7) Secondary analyses of previously trialled interventions

\subsection{Synthesis of program components and outcomes}

A logic model was developed to synthesise the components and outcomes of the interventions. Logic models are increasingly valued as a tool for evaluating complex interventions within a systematic review (Anderson et al, 2011). Elements of the Kneale, Thomas, and Harris (2015) guidelines were adopted for this iterative process, which comprised of the following:

1) Identifying the intervention programme core components and theoretical models

2) Identifying outputs: the mechanisms by which theoretical models and core components were operationalised

3) Identifying proximal significant outcomes: those which directly resulted in improved selfmanagement or reduction of risk factors 
4) Identifying intermediate significant outcomes: lifestyle modifications which could potentially lead to proximal outcomes

5) Identifying distal significant outcomes: social and psychological factors which could potentially support proximal outcomes

6) Linking proximal, intermediate, and distal outcomes to the needs of people with ID diagnosed or at risk of T2D. A meta-aggregation of the literature (Maine, Dickson, Brown, and Truesdale, 2018) provides a rigorous account of the needs of people with ID selfmanaging T2D, and was integrated into the logic model of the present review.

Each stage of identification was validated by the second reviewer.

\subsection{Quality Assessment}

This review employed the Effective Public Health Practice Project (EPHPP) Quality Assessment Tool for Quantitative Studies (2008). The tool comprises 8 components:
A. Selection bias: Representativeness of participants to the target population
B. Study design: ranging from RCT to study not described
C. Control of confounding factors: such as demographics
D. Blinding: of outcome assessors and participants
E. Data collection methods: reliability and validity (vi)
F. Withdrawals and drop-outs: attrition rates
G. Intervention integrity
H. Intervention analyses. 
The tool was developed to assess public health interventions and encompasses criteria for cross-sectional and clinical case studies. Therefore, not all assessment components were relevant to self-management interventions; "D", "G" and "F" were not utilised in the current review, in line with previous work such as Macrae et al. (2015). The remaining five criteria comprised the "component ratings", and were assigned the quality ratings of "Strong", "Moderate" or "Weak". The "Global rating" comprised of "component rating" scores including "Strong" (no "Weak" ratings), "Medium" (one "Weak" rating), "Weak" (two or more "Weak" ratings).

\subsection{Theoretical Model Evaluation}

Theoretical models were evaluated using Michie and Prestwich's (2010) Theory Coding Scheme (TCS). The TCS enables evaluation of the relevant theoretical constructs targeted by intervention techniques, such as whether they are measured, whether the intervention facilitates behaviour change, and whether or not a single theory is adopted.

\section{Results}

\subsection{Study Characteristics}


A total of 23 research articles were identified: 1) Bradshaw et al., 2007; 2) Biddle et al., 2015; 3) Contento, Koch, Lee, and Calabrese-Barton, 2010; 4) Davies et al., 2008; 5) Dutton, Provost, Tan, and Smith, 2008; 6) Faro, Ingersoll, Fiore, and Ippolito, 2005; 7) Glasgow et al., 2012; 8) Hartmann et al., 2012; 9) Heideman et al., 2015; 10) Jennings, Vandelanotte, Caperchione, and Mummery, 2014; 11) Laatikainen et al., 2007; 12) Macedo, Cortez, dos Santos, Reis, Torres, 2017; 13) McCurley et al., 2017; 14) Miller, Kristeller, Headings, and Nagaraja, 2014; 15) Mohamed, Al-Lenjawi, Amuna, Zotor, and Elmahdi, 2013; 16) Ramadas et al., 2015; 17) Sacco, Malone, Morrison, Friedman, and Wells, 2009; 18) Saksvig et al., 2005; 19) Taggart et al., 2017; 20) Thoolen, de Ridder, Bensing, Gorter, and Rutten, 2008; 21) Wu et al., 2011; 22) Yates et al., 2009; 23) Yates et al., 2016). The PRISMA diagram below, Figure 1, details the selection and elimination of studies at each stage of the review. Following this, Table 1 provides an overview of included study characteristics.

Insert Figure 1, then Table 1 here

\subsubsection{Study range}

The included studies were published between 2005 and 2017. Although the search engines produced earlier results, 2005 was the first year in which inclusion criteria was fulfilled. Earlier reviews by Brown (1990) and Norris, Engelgau, and Narayan (2001) criticize the lack of application of theoretical models in diabetes intervention programmes (Skinner, Cradock, Arundel and Graham, 1990), thus the selection criteria resulted in the selection of studies from 2005, by which point theoretical models were more clearly demonstrated. 


\subsubsection{Geographical origins of studies}

A majority of nine (39.1\%) studies were conducted in the U.S. (Bradshaw et al., 2007;

Contento et al., 2010; Dutton et al., 2008; Faro et al., 2005; Glasgow et al. 2012; Jennings et al., 2014; McCurley et al., 2017; Miller et al., 2014; Sacco et al., 2009). The remainder were published in the U.K. (five studies, 21.7\%: Biddle et al., 2015; Davies et al., 2008; Taggart et al., 2017; Yates et al., 2009; Yates et al., 2016), the Netherlands (two studies, 8.7\%:

Heideman et al., 2015; Thoolen et al., 2008), Australia (one study, 4.3\%: Laatikainen et al., 2007), China (one study, 4.3\%: Wu et al., 2011), Germany (one study, 4.3\%: Hartmann et al., 2012), Qatar (one study, 4.3\%: Mohamed et al., 2013), Malaysia (one study, 4.3\%: Ramadas et al., 2015), Brazil (one study, 4.3\%: Macedo et al., 2017), and Canada (one study, 4.3\%: Saksvig et al., 2005). The increased prevalence of U.S. studies may reflect the search platform (for instance, PsycINFO, indexes predominantly American Psychology Association research articles. The five U.K. studies were developed in collaboration with the NHS at Leicester Diabetes Centre and contain shared components of the core program (Davies et al., 2008).

\subsubsection{Sample size}

Sample size ranged from $N=27$ (Faro et al. 2005) to $N=1134$ (Contento et al., 2010), mean 254.5 .

\subsubsection{Participant age and diabetes status}

The majority $(N=19)$ of studies provided the mean age of the participants, and the mean age was 53.6 years (SD 12.4). Mean age of the samples ranged from 12 years (Contento et al., 
2010), to 64 years (Sacco et al., 2009; Yates et al., 2016). Indeed, the onset of Type 2 diabetes is more prevalent in those over 40 and can reduce life expectancy by ten years (Diabetes UK, 2017). This may account for such study characteristics.

With regard to diabetes status, the majority $(N=14.60 .9 \%)$ of studies recruited were self-management programmes and hence recruited participants diagnosed with diabetes. (Bradshaw et al., 2007; Davies et al., 2008; Dutton et al., 2008; Glasgow et al., 2012; Hartmann et al., 2012; Jennings et al., 2014; Macedo et al., 2017; Miller et al., 2014; Mohamed et al., 2013; Ramadas et al., 2015; Sacco et al., 2009; Taggart et al., 2017; Thoolen et al., 2008; Wu et al., 2011). The remaining nine (39.1\%) studies were preventative and recruited participants identified as 'at risk' of diabetes (Biddle et al., 2015; Contento et al., 2010; Faro et al., 2005; Heideman et al., 2015; Laatikainen et al., 2007; $\quad$ McCurley et al., 2017; Saksvig et al., 2005), with impaired glucose tolerance (Yates et al., 2009), and prediabetic status (Yates et al., 2016). The mean participant age of these studies was lower than that of the self-management programme studies (47.4 and 57.1 respectively), reflecting a strategy to target risk factors at a younger age. The mean age of participants with ID with or at risk of diabetes in a review by Macrae et al. (2015) was 40.06 years, suggesting that the onset of diabetes may be earlier in this population than in the mainstream population. This may be indicative of health inequalities such as people with ID being less physically active and having poorer diets. Thus, the importance of early intervention or preventative approaches for addressing diabetes in this population is apparent. As people with ID are likely to be at risk at an earlier age than this, preventative programmes should be aimed at younger adults accordingly.

\subsubsection{Gender and ethnicity}


All studies except Mohamed et al. (2013) reported gender demographics. There was a mean female participant rate of 54.9\% (SD 16.6), and mean male rate of $44.9 \%$ (SD 16.5). Gender demographics were balanced at baseline in all studies, as described further below. The current literature does not highlight the necessity of a gender focussed diabetes intervention programme for people with ID at this time.

\subsubsection{Programme delivery and structure}

Programme duration was diverse, ranging from a single three-hour session (Yates et al., 2009) to delivery over a year within an academic curriculum (Faro et al., 2006; Saksvig et al., 2005. Sixteen (69.6\%) studies were aimed at group level (Contento et al., 2010; Biddle et al., 2015; Bradshaw et al., 2009; Davies et al., 2008; Faro et al., 2005; Hartmann et al., 2012; Heideman et al., 2015; Laatikainen et al., 2007; Miller et al., 2014; Macedo et al., 2017; McCurley et al., 2017; Mohammed et al., 2013; Saksvig et al., 2005; Taggart et al., 2017; Yates et al., 2009; Yates et al., 2016); five (21.7\%) aimed at individual level (Dutton et al., 2008; Jennings et al., 2014; Ramadas et al. 2015; Sacco et al., 2009; Wu et al., 2011), and two $(8.7 \%)$ comprised both group and individual components (Glasgow et al., 2012; Thoolen et al., 2008). Group level interventions were typically structured education programmes, while those aimed at an individual level were delivered through 1-1 counselling sessions. Group based interventions may provide social support through shared learning. Further research into the psychosocial experiences of people with ID self-managing diabetes could elucidate whether or not the influence of social setting is important. A group, individual, or mixed setting could be selected on this basis.

\subsection{Impact and effectiveness: significant outcomes}


A logic model is presented below (Figure 2), which illustrates the relationship between the programme components and theoretical models, intervention techniques, significant outcomes, and the needs of people with ID. Studies are presented hierarchically in order of frequency. Numbering corresponds to the list at the start of the results section (3.1.).

Insert Figure 2 here

\subsubsection{Proximal outcomes}

Proximal outcomes were those which had a direct impact upon T2D progression risk factors, and included improvements in diet, physical activity, blood glucose levels and weight/waist circumference.

\subsubsection{Improved diet}

Improved diet was a significant outcome in five $(21.7 \%)$ studies. Two of these were T2D self-management programs (Bradshaw et al., 2007; Miller et al., 2014) and three were prevention programs (McCurley et al., 2017; Contento et al., 2010; Saksvig et al., 2005). Contento et al. 2010 and Saksvig et al. (2005) were aimed at adolescents in a secondary education setting, and the remaining studies were aimed at adults. Outcomes were achieved through an educational curriculum which taught dietary knowledge and skills, with support from primary care/specialist support, and additionally social support. The interventions were theoretically informed by SCT; SDT; Mindfulness; Ecological model; Theory of meaningful learning. Intervention techniques included using group sessions; goal setting; self- 
assessment/reflection; elicited learning; cultural tailoring; story-telling; mediation; social support through correspondence; environmental factors such as a tailored school meal and reduced access to unhealthy snacks and drinks; use of a CD ROM.

\subsubsection{Improved physical activity}

Improved physical activity was a significant outcome in three (13\%) T2D self-management studies (Bradshaw et al., 2007; Glasgow et al., 2012; Jennings et al., 2014) and two (8.7\%) preventative studies, (Yates et al. 2009; Yates et al., 2016). Yates et al., 2009 trialled a structed educational programme, "PREPARE", which aimed to increase ambulatory activity. This was delivered in a primary care setting, as was "Walking Away from Diabetes" by Yates et al. (2016). Both programs were developed from a wider diabetes educational programme called DESMOND (Davies et al., 2008), and hence shared core components, theoretical basis and outputs. Other programs had core components which reflected those of "Improved diet" above. Theoretical basis was driven by SCT; SLT; SRT; Dual process theory; Ecological model; the Theory of planned behaviour. Core components and theoretical models were operationalised using group sessions; resiliency promotion; goal setting; selfassessment/reflection; role play; internet delivered sessions; utilising pedometers; elicited learning; identifying risk factors.

\subsubsection{Improved Glucose levels}


Improving levels of blood glucose was significant in three (13\%) T2D self-management programmes (Macedo et al., 2017; Mohamed et al., 2013; Taggart et al., 2017) and one preventative (Yates et al., 2009). Taggart et al. (2017) conducted a feasibility trial adapting DESMOND (Davies et al., 2008) for people with ID. All programmes were group based educational curriculum with support from primary care/specialist support. Interventions were informed by SCT; SLT; CST; DPT; the HBM. Intervention techniques which may have led to these outcomes were diet, diabetes, and physical activity knowledge and skills; group learning; resiliency promotion; goal setting; role play; utilising pedometers; elicited learning; identifying risk factors.

\subsubsection{Reduced weight/waist circumference}

Three (13\%) studies achieved significant reductions in weight or waist circumference (Contento et al., 2010; Heideman et al., 2015; Laatikainen et al., 2007), of which all were preventative. These studies also involved educational curriculum with primary care/specialist support, and were theoretically informed by SCT; SLT; CST; DPT; SRT. Relevant intervention techniques included diet and physical activity knowledge; group learning; resiliency promotion; goal setting; self-assessment/reflection; role play; elicited learning; identifying risk factors.

\subsubsection{Intermediate outcomes}

Intermediate outcomes are those which impact upon healthy knowledge and behaviours, and subsequently have the potential to lead to or enhance the proximal outcomes described above. These included improved knowledge around diet and physical activity, and smoking cessation. 


\subsubsection{Improved dietary knowledge}

Five (21.7\%) studies yielded significant improvements in dietary knowledge (Macedo et al., 2017; Miller et al., 2014; Mohamed et al., 2013; Saksvig et al., 2005; Taggart et al., 2017). These outcomes unsurprisingly related to the programme components reported above in dietary improvements. However, the interventions were additionally informed by the Health belief model, Health action process approach and Behaviour change protocol. Intervention techniques also included goal setting and role play, which were not described in the interventions with proximal dietary improvements.

\subsubsection{Improved Physical activity knowledge}

A significant outcome in physical activity knowledge was reported in one (4.3\%) study (Taggart et al., 2017). This was not matched with a proximal outcome of increased physical activity, which was not a measured outcome. Despite this, there were shared components, theoretical models, and intervention techniques with the studies which reported significant improvements in physical activity.

\subsubsection{Smoking cessation}

Smoking cessation was reported as a significant outcome in one Davies et al. (2008), which is described above in "Improved physical activity". Other programmes with shared programmes and theoretical models, such as Taggart et al. 2017 and Yates et al. 2016 may also have yielded a significant result for smoking cessation, were this a measured outcome. 


\subsubsection{Distal outcomes}

Distal outcomes included those which could potentially enhance intermediate outcomes, thus leading to behavioural change and improvements in health. These were primarily psychosocial, and reflected the theoretical models adopted. Overall Self-efficacy was significant in five (21.7\%) studies (Bradshaw et al., 2007; Contento et al., 2010; Miller et al., 2014; Thoolen et al., 2008; Wu et al., 2011). These studies were commonly informed by SCT, in which Self-efficacy is a key construct (Nutbeam and Harris, 2010). Intervention techniques which may have led to these outcomes included resiliency training, goal setting, and self-assessment/reflection,

Related distal outcomes were dietary Self-efficacy (Saksvig et al., 2005; Taggart et al., 2017), walking Self-efficacy (Yates et al., 2009), outcome expectations (Miller et al., 2014; Wu et al., 2011), Locus of control (Brashaw et al., 2007). Illness belief score reflected the use of Leventhal's CST in Davies et al. (2008), and lower depression the use of Mindfulness in Hartman et al. (2012), and Miller et al. (2014).

\subsection{Quality Assessment}

An overview of the quality assessment process is provided in Table 2, below. The EPHPP quality assessment tool (2008) was utilised to assess the quality of the studies. The researcher's ratings were later verified by the second and third authors to ensure inter-rater reliability. In terms of the global ratings, seven (30.4\%) studies were given a "Strong" rating (Biddle et al., 2015; Contento et al., 2010; Davies et al., 2008; Hartmann et al., 2012; Heideman et al., 2015; Macedo et al., 2017; Sacco et al., 2009). The remaining majority had one or more "Weak" component ratings. Of these, 11 (47.8\%) received a "Moderate" global rating (Faro et al., 2005; Glasgow et al., 2012; Laatikainen et al., 2007; Miller et al., 2014; 
Mohammed et al., 2013; Ramadas et al., 2015; Taggart et al., 2017; Thoolen et al., 2008; Wu et al., 2011; Yates et al., 2009; Yates et al., 2016), and five (21.7\%) received a "Weak" global rating, with two or more "Weak" component ratings (Bradshaw et al., 2007; Dutton et al., 2008; Jennings et al., 2014; McCurley et al., 2017; Saksvig et al., 2005).

The "local" category which contained the most "weak" scores was "Representativeness" (12 studies, 52.2\%). This was due to an absence of the description of the number of invited participants who continued to participate in the studies, or to the low number of participation after invitation. However, it should be noted that where powered samples required a high number of participants (such as Davies et al., 2008, Yates et al., 2009, and Yates et al., 2016), these sample targets were achieved, despite the low invited participant percentage (example for $20.7 \%$ in Yates et al., 2016). Nine (39.1\%) studies used a powered sample calculation. In these studies, it is apparent that the recruitment drive required a very large, multiple-site approach, therefore the "Representativeness" category limited as an indicator of quality in these examples.

Insert Table 2 here

\subsection{Theoretical Model Evaluation}

Details of the Michie and Prestwich (2010) Theory Coding Scheme (TCS) evaluations are provided in Table 3. There were two studies (8.7\%) which met all of the TSC criteria (Miller et al., 2014; Wu et al., 2011). Five studies (21.8\%) were rated 5/6 (Contento et al., 2010; 
Hartmann et al., 2012; Heideman et al., 2015; Sacco et al., 2009; Thoolen et al., 2008). The remaining studies met $4 / 6$ of the criteria or less.

All of the studies explicitly specified use of a theoretical model in the introduction or abstract, with the exception of Bradshaw et al. (2007), who described Self-efficacy constructs but did not provide the wider theoretical model. The majority of the studies $(\mathrm{N}=17,73.9 \%)$ provided evidence for the efficacy of theoretical constructs.

Fewer studies used a single theoretical model to inform an intervention $(\mathrm{N}=6,26 \%)$. Michie and Prestwich (2010), suggest the efficacy of multiple model-based interventions can be unclear as it is not possible to tell which construct has affected an outcome. Therefore, theoretical comparisons cannot be made. Systematic reviews and meta-analyses (such as Gourlan et al., 2016) comparing the efficacy of single versus multiple theory-based interventions, have reported a higher impact on physical activity from single-theory interventions. However, the trend of multi-component interventions in the studies selected in the present review suggests that single model-based interventions may not be appropriate for addressing behavioural change in type 2 diabetes self-management, despite the difficulty of evaluating the efficacy of multiple-theoretical approaches.

In terms of the frequency of models, whether as standalone or alongside others, there were models which were use more recurrently than others. SCT was used in $15(65.2 \%)$ multi-model studies and one single-model. The five U.K. studies were based on a core programme (Davies et al., 2008) and shared SCT, SLT, Leventhal's CSM, and DPT.

The construct most explicitly described as informing intervention technique was Selfefficacy, which was used to inform intervention techniques in $10(43.5 \%)$ studies. These techniques were associated with a range of successful outcomes, including physical activity (Yates et al., 2009), diet (Miller et al., 2014), and diabetes knowledge (Sacco et al. 2009). 
The prevalence of Self-efficacy reflects on its practical applicability as a construct within SCT and also the Transtheoretical model (Nutbeam and Harris, 2010).

The majority of the studies did not meet the criteria "Evaluation of theory and constructs in discussion", with exception of six (26\%), (Bradshaw et al., 2007; Miller et al. 2014; Sacco et al. 2009; Saksvig et al., 2005; Thoolen et al., 2008; Wu et al., 2011). These evaluations related to the promotion of Self-efficacy for self-directed care (Bradshaw et al., 2007; Wu et al., 2011), the usefulness of Mindfulness as a measure for regulating eating behaviours alongside diabetes self-management education (Miller et al. 2014), the potential for lower depression when correlated with higher Self-efficacy (Sacco et al., 2009), and the application of proactive coping to self-management. These did not discuss whether or not models required further adaptation, and were limited to advocating the models for future use.

From these findings and the logic model presented in figure 2 above, it can be concluded that there are several consistently applied theoretical models which have led to significant distal outcomes, thereby furthering the protentional for behavioural change. However, application of many models is limited due to the lack of clear description of how intervention techniques were informed by theoretical constructs, and of what the implications for models would be following outcome. The use of multiple-theories also limited the clarity of efficacy as single theories could not be examined in isolation. However, the frequency and diversity of the application of the Self-efficacy construct highlight its potential for consideration alongside the needs of people with ID self-managing T2D.

Insert Table 3 here

\section{Discussion}




\subsection{Study Characteristics}

The primary aim of this review was to ascertain the characteristics of theory-based T2D intervention programs and evaluate these in relation to the needs of people with ID. The findings demonstrate that the mean age in the studies extracted for the present review (mean 57.1 years in T2D self-management studies and 47.4 I years in preventative) is higher than that of the participants with ID in the studies highlighted in Mcrae et al. (2015), which was 40.06 years. The studies in Mcrae et al., (2015) and McVilly et al. (2014) highlighted that people with ID experienced frustration in adjusting to self-management behaviours, therefore there is a rationale for introducing preventative diabetes education at an earlier age in order to reduce the need for difficult adjustment later in life.

Dabelea et al., (2014) reported that the number of youth diagnosed with T2D increased by more than $30 \%$ from 2000 to 2009 suggesting that it is no longer primarily a condition of older adults, and TD2 developed during childhood is more likely to result in higher morbidity and mortality rates than in adulthood. This highlights the importance of early intervention and preventative measures for people with and at risk of diabetes. Furthermore, a review of pharmacological and lifestyle interventions to prevent or delay T2D found that $80-90 \%$ of all cases of T2D could be prevented by targeting lifestyle factors using structured education programmes (Gillies et al., 2007). There were nine (39.1\%) preventative studies identified in the present review (Biddle et al., 2015; Contento et al., 2010; Faro et al., 2005; Heideman et al., 2015; McCurley et al., 2017; Laatikainen et al., 2007; Saksvig et al., 2005; Yates et al., 2009; Yates et al., 2016). The characteristics of these studies may be of particular relevance for the development of interventions aimed at people with ID. The potential to reduce diabetes related costs through lifestyle interventions is estimated at 40- 
$60 \%$ (Gillies et al., 2007). However, given that the additional support requirements of T2D self-management for PWID are likely to be higher than that of the general population, preventative approaches through risk factor reduction may come to be seen as more costeffective. The 3-hour structured educational programs used in Biddle et al. (2015) and Yates et al (2009 and 2016) have been identified as low cost (National Institute for Heath and Care Excellence), and are therefore resource-efficient.

As illustrated by the logic model in Figure 2, the significant proximal, intermediate, and distal outcomes align to the needs of people with ID, as identified by a meta-aggregation of the needs of people with ID self-managing T2D (Maine et al., 2018). The metaaggregation identified that the people with ID's understanding of T2D was limited. It was highlighted in Cardol et al. (2012a) for example, that whilst some people with ID worried about the consequences of T2D, others did not "feel ill", and only perceived diabetes as serious when insulin injections were involved. In terms of improving this understanding, programmes which utilized Self-regulation theory (SRT) may be suitable, as these encouraged participants to reflect on and re-evaluate their current understanding within educational sessions. Additional techniques such as elicited learning, role play and storytelling, may also have aided learning and could potentially be used for people with ID. Studies which employed the DESMOND curriculum (Biddle et al., 2015; Davies et al., 2008; Taggart et al., 2017; Yates et al., 2009; Yates et al., 2016) utilised this model and intervention techniques, and this may have contributed to the success of Taggart et al.'s (2017) adaptation of DESMOND for people with ID. Participants in the process evaluation focus groups within this study described being able to understand the content, which therefore suggests that the program sufficiently addressed this need. In addition to SRT, the Dual process theory (DPT) may aid people with ID's understanding through elicited, non-didactic learning, which was also successfully employed by Taggart et al. (2017). 
It has been highlighted that existing T2D resources for people with ID are not appropriate. For example, caregivers in Hale et al. (2011) reported that any available resources were not in an accessible format. Detailed information on the format of resources in the studies identified in the present review was not included in the research papers. However, it is likely that further modifications may be required, such as pictorial support and accessible language. The DESMOND-ID adaptation by Taggart et al. (2017) used modified resources, and furthermore delivery was provided with support from ID nurses, who may have been able to tailor materials and contents accordingly.

Another need identified by the meta-aggregation (Maine et al., 2018) was in emotional support regarding lifestyle adjustments. Participants with ID reported experiencing frustration around dietary and medication routines, and struggled with adherence (Dysch et al., 2012, Hale et al., 2005; Rey-Conde et al., 2005). Hartmann et al. (2012), and Miller et al. (2014) targeted diabetes related distress using Mindfulness-based meditation. A systematic review by Chapman et al. (2013) found evidence for the efficacy of Mindfulness for people with ID in the areas of aggression and sexual arousal, though the evidence base was limited due to methodological inconsistencies. However, Mindfulness may be a supportive tool for people with ID self-managing T2D.

A common approach across the studies identified in the present review was improving participants' beliefs in their ability to self-manage or reduce risks of developing T2D. The theoretical basis for this was SCT, utilising self-efficacy enhancing techniques such as goal setting, positive feedback, self-assessment/reflection, resiliency promotion, and social support. With regard to people with ID, these techniques may lead to a reduction in struggling to adhere to healthy lifestyle changes, and coping with barriers such as living with others who do not have dietary restrictions. The Self-efficacy enhancing mechanism, "Vicarious experiences" (Bandura, 1977), advocates learning from the successful experiences 
of peers. The majority of the programs were delivered in a group setting $(18 / 23,78.3 \%)$, offering opportunities for social modelling. Additionally, the environment may have played a role in enhancing Self-efficacy. Saksvig et al. (2005) and Faro et al. (2005) delivered programmes in a school setting over an academic term, and additionally involved parents and carers of students through correspondence. This may have contributed to a significant improvement in dietary habits and dietary self-efficacy through environmental and social opportunities for reinforcement of key-messages. With regard to people with ID, Maine et al. (2017) reported that the Self-efficacy construct may be appropriate for people with ID selfmanaging T2D, but that social support is required for meaningful self-reflection. An environment which provides this support may therefore be of paramount importance.

Pedometers were used to measure ambulatory in three studies (Bradshaw et al., 2007; Biddle et al., 2015, Yates et al., 2009). As well as a measure, wearing pedometers may have led to increases in physical activity; indeed Yates et al. (2009) yielded significant changes in ambulatory activity in the pedometer group only, which was attributed to the pedometers providing ongoing feedback and achieving goals, thus enhancing Self-efficacy. Pedometers have been used successfully to measure ambulatory activity in people with ID (Temple and Stanish, 2009), and this may therefore be an important component in programmes for people with ID which aim to increase physical activity and reduce sedentary behaviour.

Limited training and knowledge in staff was identified as a theme in the metaaggregation carried out by Maine et al. (2018), which further highlights the need for the inclusion of social support components in potential interventions and prevention programs. Whilst social support was a common approach across the studies in the present review, the support network of participants was not always clearly delineated. By virtue of having a disability, people with ID who are approached to participate in research are more likely to have access to support, which could potentially be incorporated into intervention or 
prevention programmes. Taggart et al. (2017) invited carers/family members to attend alongside people with ID, and also to an additional three-hour session on their own, which was met by a $94 \%$ attendance rate. The trial protocol of a second T2D self-management programme (Walwyn et al., 2015), also invites caregivers/family members to attend sessions. The development report of a third programme, STOP Diabetes, also involved carers in the pilot stages (Dunkley et al., 2017). A programme of one carer session followed by seven 2.5h sessions over 7 weeks was reported as acceptable to participants. These studies therefore address the social support needs identified in the literature. As described above, the delivery environment may also be an important factor, and an educational setting, as utilised by Saksvig et al. (2005) and Faro et al. (2005), may provide a support and reinforcement so the key messages can be sustained over time. Recruitment of people with ID in further educational colleges or special schools for people with ID is worth further exploration. The discussion now turns to the quality evaluation of the studies selected in this review.

\subsection{Quality Assessment}

The second aim of this review was to systematically appraise the quality of T2D intervention studies using the EPHPP (2008) guidelines. There was not a consistently high quality across the studies, mainly due to the lack of representativeness of the studies. This reflected on the purposeful sampling of the studies, where participants were selected from existing groups for convenience. The low percentage of invited participants who agreed to participate also led to there being few "strong" ratings under this criterion. Representativeness is also likely to be an issue a study for recruitment of people with ID diagnosed or at risk of T2D, and this criterion may therefore not be a useful quality indicator. 


\subsection{Theoretical Application}

The final aim of this review was to evaluate the theoretical models used in mainstream T2D interventions in terms of clarity of application. As with the EPHPP evaluation, quality was not consistently high, and whilst this could also arguably be due to the suitability of criteria such as the advocacy of single models, the application of model constructs to intervention techniques remains unclear.

Social Cognitive Theory (SCT) was found to be the most clearly evidenced model, and the SCT construct, Self-efficacy (Bandura, 1977), was associated with diabetes knowledge, physical activity, and dietary behaviours and thus provided several significant outcomes, as described above. SCT is based on the learning relationship between the individual and their social context (Bandura 1977), emphasising the importance of the learning environment. Behavioural change can come about through reciprocal determinism, a process in which the individual and environment influence each other. Social norms, the behaviour of peers (termed "vicarious experiences") and the persuasive influence of others (termed "verbal persuasion") shape the cognition of an individual (Nutbeam and Harris, 2010). For people with ID, whose learning environment is often restricted to home, day and residential care settings, socially-produced behavioural change may be a greater challenge than for the general population. In terms of "verbal persuasion", the values, attitudes and care approaches of family members and support workers often determine the level of autonomy people with ID have in self-managing their T2D and thus determine their level of opportunity for learning (Trip et al., 2015 and Whitehead et al., 2016). "Vicarious" learning experiences may be similarly restricted as PWID often live together in shared care settings on the basis of their disability rather than their diabetes status. Hale et al. (2011) reported a sense of 'unfairness' by those with T2D who compare themselves to the non-disabled with T2D regarding issues such as dietary restrictions and medication regimes. SCT-based 
interventions aimed at people with ID self-managing T2D should therefore take into account the complexities of peer comparisons and social support in relation to the learning environment.

The second component of SCT is Outcome Expectations, which focuses on the perceived consequences of behaviours, such as reducing the risk of cardiovascular complications because of poor diet and lack of physical activity (Nutbeam and Harris, 2010). Short term outcomes can be more straight-forward to understand than long term to those with ID, necessitating small, manageable goals and steps. Hale et al. (2011) and Dysch et al. (2012) highlighted that people with ID can struggle to understand the long-term consequences of poor diabetes control such as blindness, renal failure and amputation. Goal setting which emphasises short-term health benefits may be of key importance to sustaining self-management behaviours over time, thereby reducing the possibility of long term and avoidable complications.

The final component of SCT is Self-efficacy, the belief in the ability to successfully perform a behaviour (Bandura, 1977). This component of the model reflects the level of effort put into a task to effect change and is viewed as the most important SCT component (Nutbeam and Harris, 2010). Bandura proposed four sources of efficacy enhancing experiences; "Mastery of Experiences", which describes the importance of successful past experiences, "Vicarious Experiences and Verbal Persuasion" (discussed above) and "Physical/Emotional Arousal", which describes the importance of physiological feedback. The model was clearly delineated and successfully used in the study by Wu et al., (2011), who alongside Miller et al. (2014) received the highest TCS ratings in the present review. The Four Sources model has not been applied to PWID self-managing T2D, and further exploration of its appropriateness should be the focus of future intervention studies aimed at this population. 


\section{Strengths and Limitations}

This review has taken a rigorous approach to identifying and evaluating theoretically driven T2D prevention and intervention programmes, with a view to identifying potential areas for adaptation to the ID population. A rigorous search strategy was used which identified studies ranging in outcomes, methodology, and design quality. The use of the EPHPP quality assessment tool (2008) enabled a systematic and contemporary critique which can be used to inform subsequent studies.

A limitation was apparent in the search criteria around diabetes. Searching specifically for T2D excluded many physical activity studies, which may have provided further insight into application of theoretical models. However, as the majority of the studies in this paper were aimed at lifestyle change through increased physical activity or improving health literacy, it is likely that the models would have been similar. Therefore, by focussing on T2D studies, those relevant to the review questions were identified and analysed.

The inclusion criteria of explicitly described theoretical models may also have limited the scope of the review, as there may have been studies which successfully targeted a range of outcomes without explicit recourse to models. However, it was important to establish the dominant and successfully applied models, so that these could be evaluated alongside the needs of people with ID who are diagnosed with or at risk of T2D as such models have not been applied to this population.

\section{Conclusions}


Over the 12-year period focussed upon in this review, an internationally diverse range of prevention and self-management programmes were identified. These were critically analysed in the context of stringent criteria. The educational needs of people with ID at risk of or selfmanaging T2D may be met through existing programmes, although further modifications may be required for print-based resources to ensure accessibility. Facilitated self-reflection and elicited learning, underpinned by self-regulation theory and dual processing theory, may enable people with ID to reflect on and modify their current understanding of T2D risk factors and consequences. Further support for diabetes-related emotional distress in people with ID could potentially be provided using mindfulness. Goal setting and resiliency training, underpinned by SCT and delivered in a peer-shared environment, may enhance selfefficacy.

This review provides support for further randomized controlled trials of programmes for people with ID under development or having undergone feasibility studies. Rationale is also provided for the development/adaptation of a preventative programme based on similar components and theoretical models. This may achieve greater impact if delivered to younger adults or adolescents with ID, and an educational setting may provide an enhanced level of social support and reinforcement, thus leading to sustained behavioural change.

\section{References}

Absetz, P., Valve, R., Oldenburg, B., Heinonen, H., Nissinen, A., Fogelholm, M., ... Uutela, A. (2007). Type 2 diabetes prevention in the "real world." Diabetes Care, 30(10), 2465-2470. doi:10.2337/dc07-0171.Clinical 
Ajzen, I (1991). "The theory of planned behavior". Organizational Behavior and Human Decision Processes 50 (2): 179-211

Ajzen, I. (1987). Attitudes, Traits, and Actions: Dispositional Prediction of Behavior in Personality and Social Psychology. Advances in Experimental Social Psychology, 20, 1-63. doi:10.1016/S0065-2601(08)60411-6

Anderson LM, Petticrew M, Rehfuess E, Armstong R, Ueffing E, Baker P, Francis D,

Tugwell D: Using logic models to capture complexity in systematic reviews. Res Synth Meth $2011,2: 33-42$.

Bandura, A. (1977). Self-efficacy: Toward a unifying theory of behavioral change. Psychological Review, 84(2), 191-215

Becker, M.H. (1974) The Health Belief Model and Personal Health Behaviour. Health Education Monographs. Vol. 2 No. 4. 153-154

Biddle, S. J. H., Edwardson, C. L., Wilmot, E. G., Yates, T., Gorely, T., Bodicoat, D. H., Davies, M. J. (2015). A Randomised Controlled Trial to Reduce Sedentary Time in Young Adults at Risk of Type 2 Diabetes Mellitus: Project STAND (Sedentary Time ANd Diabetes). Plos One, 10(12), e0143398. doi:10.1371/journal.pone.0143398

Bradshaw, B. G., Richardson, G. E., Kumpfer, K., Carlson, J., Stanchfield, J., Overall, J. Kulkarni, K. (2007). Determining the efficacy of a resiliency training approach in adults with type 2 diabetes. The Diabetes Educator, 33(4), 650-659. doi:10.1177/0145721707303809

Brown, S.A. (1990). Studies of educational interventions and outcomes in diabetic adults: a meta-analysis revisited. Patient Educ Couns 16:189-215, 1990 
Cardol, M., Rijken, M., \& van Schrojenstein Lantman-de Valk, H. (2012a). Attitudes and dilemmas of caregivers supporting people with intellectual disabilities who have diabetes. Patient Education and Counseling, 87(3), 383-388. doi:10.1016/j.pec.2011.11.010

Cardol, M., Rijken, M., \& van Schrojenstein Lantman-de Valk, H. (2012b). People with mild to moderate intellectual disability talking about their diabetes and how they manage. Journal of Intellectual Disability Research, 56(4), 351-360. doi:10.1111/j.13652788.2011.01472.x

Chapman, M. J., Hare, D. J., Caton, S., Donalds, D., McInnis, E., \& Mitchell, D. (2013). The Use of Mindfulness with People with Intellectual Disabilities: a Systematic Review and Narrative Analysis. Mindfulness, 4(2), 179-189. http://doi.org/10.1007/s12671-013$\underline{0197-7}$

Contento, I. R., Koch, P. A., Lee, H., \& Calabrese-Barton, A. (2010). Adolescents demonstrate improvement in obesity risk behaviors after completion of choice, control \& change, a curriculum addressing personal agency and autonomous motivation. Journal of the American Dietetic Association, 110(12), 1830-9. doi:10.1016/j.jada.2010.09.015

Davies, M. J., Heller, S., Skinner, T. C., Campbell, M. J., Carey, M. E., Cradock, S., Khunti, K. (2008). Effectiveness of the diabetes education and self-management for ongoing and newly diagnosed (DESMOND) programme for people with newly diagnosed type 2 diabetes: cluster randomised controlled trial. British Medical Journal (Clinical Research Ed.), 336(7642), 491-5. doi:10.1136/bmj.39474.922025.BE 
Dabelea, D., Mayer-Davis, E. J., Saydah, S., Imperatore, G., Linder, B., Divers, J., Hamman, R. F. (2014). Prevalence of type 1 and type 2 diabetes among children and adolescents from 2001 to 2009. Jama, 311(17), 1778-86. doi:10.1001/jama.2014.3201

Dunkley, A. J., Tyrer, F., Doherty, Y., Patel, N., \& Spong, R. (2017). Development of a multi-component lifestyle intervention for preventing type 2 diabetes and cardiovascular risk factors in adults with intellectual disabilities, (January), 1-10. http://doi.org/10.1093/pubmed/fdx067

Dutton, G. R., Provost, B. C., Tan, F., \& Smith, D. (2008). A tailored print-based physical activity intervention for patients with type 2 diabetes. Preventive Medicine, 47(4), 409-11. doi:10.1016/j.ypmed.2008.06.016

Dysch, C., Chung, M. C., \& Fox, J. (2012). How do people with intellectual disabilities and diabetes experience and perceive their illness? Journal of Applied Research in Intellectual Disabilities, 25, 39-49. doi:10.1111/j.1468-3148.2011.00641.x

Emerson, E. (2005) Underweight, obesity and exercise among adults with intellectual disabilities in supported accommodation in Northern England. Journal of Intellectual Disability Research Volume 49 Part 2 pp 134-143

Emerson, E. and Baines, S., (2010) Health Inequalities \& People with Learning Disabilities in the UK: 2010, Learning Disabilities Observatory Retrieved from https://www.improvinghealthandlives.org.uk

Faro, B., Ingersoll, G., Fiore, H., \& Ippolito, K. S. (2005). Improving Students’ Diabetes Management Through School-based Diabetes Care. Journal of Pediatric Health Care, 19(5), 301-308. doi:10.1016/j.pedhc.2005.03.004 
Gillies, C. L., Abrams, K. R., Lambert, P. C., Cooper, N. J., Sutton, A. J., Hsu, R. T., \& Khunti, K. (2007). Pharmacological and lifestyle interventions to prevent or delay type 2 diabetes in people with impaired glucose tolerance: systematic review and meta-analysis. British Medical Journal, 334(7588), 299-299. doi:10.1136/bmj.39063.689375.55

Glasgow, Wang, L., Thakur, V. V, Leung, C. S., Tirado-, J., Bailey, C. M., ... William, L. (2012). NIH Public Access, 130(29), 9492-9499. doi:10.1021/ja8019214.

Gourlan, P. Bernard, C. Bortolon, A. J. Romain, O. Lareyre, M. Carayol, G. Ninot \& J. Boiché (2016). Efficacy of theory-based interventions to promote physical activity. A meta-analysis of randomised controlled trials, Health Psychology Review, 10:1, 5066, DOI:10.1080/17437199.2014.981777

Hale, Leigh A.; Trip, Henrietta T.; Whitehead, Lisa; Conder, Jenny; (2011) Self-management abilities of diabetes in people with an intellectual disability living in New Zealand. Journal of Policy and Practice in Intellectual Disabilities, Vol 8(4), Dec, 2011. pp. $223-230$

Hartmann, M., Kopf, S., Kircher, C., Faude-Lang, V., Djuric, Z., Augstein, F., Nawroth, P. P. (2012). Sustained Effects of a Mindfulness-Based. Diabetes Care, 35(5), 11-13. doi:10.2337/dc11-1343.

Heideman, W. H., de Wit, M., Middelkoop, B. J., Nierkens, V., Stronks, K., Verhoeff, A. P., \& Snoek, F. J. (2015). Diabetes risk reduction in overweight first degree relatives of type 2 diabetes patients: Effects of a low-intensive lifestyle education program (DiAlert) A randomized controlled trial. Patient Educational and Counselling, 98(4), 476-483. doi:10.1016/j.pec.2014.12.008 
Hex, N., Bartlett, C., Wright, D., Taylor, M., \& Varley, D. (2012). Estimating the current and future costs of Type 1 and Type 2 diabetes in the UK, including direct health costs and indirect societal and productivity costs. Diabetic Medicine, 29(7), 855-862. doi:10.1111/j.1464-5491.2012.03698.x

Heideman, W. H., de Wit, M., Middelkoop, B. J., Nierkens, V., Stronks, K., Verhoeff, A. P., \& Snoek, F. J. (2015). Diabetes risk reduction in overweight first degree relatives of type 2 diabetes patients: Effects of a low-intensive lifestyle education program (DiAlert) A randomized controlled trial. Patient Educational and Counselling, 98(4), 476-483. doi:10.1016/j.pec.2014.12.008

Jennings, C. a., Vandelanotte, C., Caperchione, C. M., \& Mummery, W. K. (2014). Effectiveness of a web-based physical activity intervention for adults with Type 2 diabetes-A randomised controlled trial. Preventive Medicine, 60, 33-40. doi:10.1016/j.ypmed.2013.12.011

Jenum, A. K., Anderssen, S. a., Birkeland, K. I., Holme, I., Graff-Iversen, S., Lorentzen, C., ... Bahr, R. (2006). Promoting physical activity in a low-income multiethnic district: Effects of a community intervention study to reduce risk factors for type 2 diabetes and cardiovascular disease: A community intervention reducing inactivity. Diabetes Care, 29(7), 1605-1612. doi:10.2337/dc05-1587

Kneale, D., Thomas, J., \& Harris, K. (2015). Developing and optimising the use of logic models in systematic reviews: Exploring practice and good practice in the use of programme theory in reviews. PLoS ONE, 10(11), 1-26.

http://doi.org/10.1371/journal.pone.0142187

Laatikainen, T., Dunbar, J. a, Chapman, A., Kilkkinen, A., Vartiainen, E., Heistaro, S., Janus, E. D. (2007). Prevention of type 2 diabetes by lifestyle intervention in an 
Australian primary health care setting: Greater Green Triangle (GGT) Diabetes Prevention Project. BMC Public Health, 7, 249. doi:10.1186/1471-2458-7-249

Liberati, A., Altman, D. G., Tetzlaff, J., Mulrow, C., Gøtzsche, P. C., Ioannidis, J. P. A., ... Moher, D. (2009). The PRISMA statement for reporting systematic reviews and metaanalyses of studies that evaluate health care interventions: Explanation and elaboration. PLoS Medicine, 6, e1000100. doi:10.1371/journal.pmed.1000100

Macedo, M. M., Noriega, D., Dos-Santos, J., Reis, A., \& De Carvalho, H. (2017). Adherence to self-care practices and empowerment of people with diabetes mellitus: a randomized clinical trial. Rev Esc Enferm USP, 51(e03278), 1-8. doi:http://dx.doi.org/10.1590/S1980-220X2016050303278

Maine, A., Dickson, A., Truesdale, M., \& Brown, M. (2017). Research in Developmental Disabilities An application of Bandura's "Four Sources of Self-Efficacy" to the selfmanagement of type 2 diabetes in people with intellectual disability : An inductive and deductive thematic analysis. Research in Developmental Disabilities, 70(April), 75-84. http://doi.org/10.1016/j.ridd.2017.09.004

Maine, A., Dickson, A., Brown, M., \& Truesdale, M. (2018). The experience of type 2 diabetes self-management in adults with intellectual disabilities and their caregivers: A review of the literature using meta-aggregative synthesis and an appraisal of rigor. Journal of Intellectual Disabilities http://doi.org/10.1177/1744629518774172

Macrae, S., Brown, M., Karatzias, T., Taggart, L., Truesdale-Kennedy, M., Walley, R., Davies, M. (2015). Diabetes in people with intellectual disabilities: A systematic review of the literature. Research in Developmental Disabilities, 47, 352-374. doi:10.1016/j.ridd.2015.10.003 
McCurley, J. L., Fortmann, A. L., Gutierrez, A. P., Gonzalez, P., Euyoque, J., Clark, T., ... Gallo, L. C. (2017). Pilot Test of a Culturally Appropriate Diabetes Prevention Intervention for At-Risk Latina Women. The Diabetes Educator, 43(6), 631-640. http://doi.org/10.1177/0145721717738020

McVilly, K., McGillivray, J., Curtis, A., Lehmann, J., Morrish, L., \& Speight, J. (2014). Diabetes in people with an intellectual disability: a systematic review of prevalence, incidence and impact. Diabetic Medicine, 31(8), 897-904. doi:10.1111/dme.12494

Melville, C. A., Cooper, S. A., Morrison, J., Smiley, E., Allan, L., Jackson, A. Mantry, D. (2008). The prevalence and incidence of mental ill-health in adults with autism and intellectual disabilities. Journal of Autism and Developmental Disorders, 38(9), 1676-1688. doi:10.1007/s10803-008-0549-7

Michie, S and Prestwich, A. (2010) Are interventions theory-based? Development of a Theory Coding Scheme. Health Psychology Review, 29 (1). 1 - 8 (8).

Miller, C. K., Kristeller, J. L., Headings, A., \&Nagaraja, H. (2014). Comparison of a Mindful Eating Intervention to a Diabetes Self-Management Intervention Among Adults With Type 2 Diabetes: A Randomized Controlled Trial. Health Education \&Behavior, 41(2), 145-154. doi:10.1177/1090198113493092

Mohamed, H., Al-Lenjawi, B., Amuna, P., Zotor, F., \&Elmahdi, H. (2013). Culturally sensitive patient-centred educational programme for self-management of type 2 diabetes: a randomized controlled trial. Primary Care Diabetes, 7(3), 199-206. doi:10.1016/j.pcd.2013.05.002

Norris, S. L., Engelgau, M. M., \& Venkat Narayan, K. M. (2001). Effectiveness of selfmanagement training in type 2 diabetes: A systematic review of randomized 
controlled trials. Diabetes Care, 24(3), 561-587.

http://doi.org/10.2337/diacare.24.3.561

Nutbeam, D., and Harris, E. (2010), Theory in a nutshell: a practical guide to health promotion theories, 3rd edition, McGraw-Hill Australia, North Ryde, NSW

O’Hara, C. (1996). Changing for Good. Journal of Cardiopulmonary Rehabilitation, 16(6), 423,424. doi10.1097/00008483-199611000-00021

Ramadas, A., Chan, C. K. Y., Oldenburg, B., Hussien, Z., \& Quek, K. F. (2015). A WebBased Dietary Intervention for People with Type 2 Diabetes: Development, Implementation, and Evaluation. International Journal of Behavioral Medicine, 22(3), 365-373. http://doi.org/10.1007/s12529-014-9445-Z

Rey-Conde, T., Lennox, N., \& McPhee, J. (2005). Diabetes and Intellectual Disability: Perceptions from People with Disability and Their Supporters. Unknown Journal Title, Unknown Vo. Retrieved from http://www.biomedsearch.com/sci/Diabetes-IntellectualDisability-Perceptions-from/0036597536.html

Sacco, W. P., Malone, J. I., Morrison, A. D., Friedman, A., \& Wells, K. (2009). Effect of a brief, regular telephone intervention by paraprofessionals for type 2 diabetes. Journal of Behavioral Medicine, 32(4), 349-359. doi:10.1007/s10865-009-9209-4

Saksvig, B. I., Gittelsohn, J., Harris, S. B., Hanley, A. J. G., Valente, T. W., \& Zinman, B. (2005). A pilot school-based healthy eating and physical activity intervention improves diet, food knowledge, and self-efficacy for native Canadian children. The Journal of Nutrition, 135(10), 2392-2398. doi:135/10/2392 [pii] ET - 2005/09/24

Scottish Public Health Observatory, 2013: Retrieved from http://www.scotpho.org.uk 
Skinner, T. C., Cradock, S., Arundel, F., \& Graham, W. (2003). Four theories and a philosophy: self-management education for individuals newly diagnosed with type 2 diabetes. Retrieved from

http://spectrum.diabetesjournals.org/cgi/content/extract/16/2/75? $\operatorname{maxtoshow}=\& H I T S$ $=10 \&$ hits $=10 \&$ RESULTFORMAT $=\&$ searchid $=1138106257613 \quad 101 \&$ FIRSTINDEX $=0 \&$ sortspec $=$ relevance $\&$ volume $=16 \&$ firstpage $=75 \&$ journalcode $=$ diaspect

Taggart, L., Coates, V., Clarke, M., Bunting, B., Davies, M., Carey, M., ... Karatzias, T. (2015). A study protocol for a pilot randomised trial of a structured education programme for the self-management of Type 2 diabetes for adults with intellectual disabilities. Trials, 16(1), 148. doi:10.1186/s13063-015-0644-y

Taggart, L., Truesdale, M., Carey, M. E., Martin-Stacey, L., Scott, J., Bunting, B., Coates, V., Brown, M., Karatzias, T., Northway R. \& Clarke, J.M. (2017) Research: Educational and psychological aspects Pilot feasibility study examining a structured selfmanagement diabetes education programme, DESMOND-ID, targeting HbA1c in adults with intellectual disabilities. Diabetic Medicine doi: 10.1111/dme.13539

Trip, H., Conder, J., Hale, L., \& Whitehead, L. (2015). The role of key workers in supporting people with intellectual disability in the self-management of their diabetes: a qualitative New Zealand study. Health \& Social Care in the Community, n/a-n/a. http://doi.org/10.1111/hsc. 12262

Tacconelli, E. (2010). Systematic reviews: CRD's guidance for undertaking reviews in health care. The Lancet Infectious Diseases (Vol. 10). doi:10.1016/S1473-3099(10)70065-7

Thoolen, B., de Ridder, D., Bensing, J., Gorter, K., \& Rutten, G. (2008). Beyond Good Intentions: the development and evaluation of a proactive self-management course for 
patients recently diagnosed with Type 2 diabetes. Health Education Research, 23(1), 53-61. doi:10.1093/her/cyl160

Utz, S. W., Williams, I. C., Jones, R., Hinton, I., Alexander, G., Yan, G., ... Oliver, M. N. (2008). Culturally Tailored Intervention for Rural African Americans With Type 2 Diabetes. The Diabetes Educator, 34(5), 854-865. doi:10.1177/0145721708323642

Walwyn, R. E., Russell, A. M., Bryant, L. D., Farrin, A. J., Wright-Hughes, A. M., Graham, E. H., ... House, A. O. (2015). Supported self-management for adults with type 2 diabetes and a learning disability (OK-Diabetes): study protocol for a randomised controlled feasibility trial. Trials, 16, 342. http://doi.org/10.1186/s13063-015-0832-9

Whitehead, L. C., Trip, H. T., Hale, L. A., \& Conder, J. (2016). Negotiated autonomy in diabetes self-management: The experiences of adults with intellectual disability and their support workers. Journal of Intellectual Disability Research, 60(4), 389-397. http://doi.org/10.1111/jir.12257

WHO: Global report on diabetes Geneva: World Health Organization. Retrieved on 3/5/16 from http://www.who.int/diabetes/global-report/en/.

Wu, S.-F. V., Lee, M.-C., Liang, S.-Y., Lu, Y.-Y., Wang, T.-J., \& Tung, H.-H. (2011). Effectiveness of a self-efficacy program for persons with diabetes: A randomized controlled trial. Nursing \& Health Sciences, no-no. doi:10.1111/j.14422018.2011.00625.x

Yates, T., Davies, M., Gorley, T., Bull, F., \& Khunti, K. (2009). Effectiveness of a Pragmatic Education Program Designed to Promote ... Diabetes Care, 32, 1404-1410. http://doi.org/10.2337/dc09-0130. 
Yates, T., Edwardson, C. L., Henson, J., Gray, L. J., Ashra, N. B., Troughton, J., ... Davies, M. J. (2016). Research : Educational and Psychological Aspects Walking Away from Type 2 diabetes : a cluster randomized controlled trial, 1-10. http://doi.org/10.1111/dme.13254 